\title{
DYNAMICS OF BODY MASS INDEX OF CHILDREN 5-7 YEARS OLD
}

\author{
Ts. Traykova* \\ Theory of the Physical Education Department, National Sports Academy „Vasil Levsky“, Sofia, \\ Bulgaria
}

\begin{abstract}
The aim of our investigation was to establish the dynamics of Body Mass index (BMI) of preschool children as a result of an enriched physical activity program. 208 children were included in the investigation.

Methods used: Theoretical analysis of literature, pedagogical experiment, anthropometry, statistical methods

Results: Children with BMI in normal levels have the highest percentage, which range from 56.2 to $72.4 \%$ for 5 -year-old boys, from 70.6 to $81.8 \%$ for the girls of the same age, from 65.8 to $84.3 \%$ for the boys 6 years old and from 57.2 to $65.4 \%$ for the girls of the same age. A high percentage of the overweight and obese children in all groups was also found. The 6-year-old girls from the experimental group have the highest BMI values $-38.5 \%$, followed by the 5-year old boys from the control group. Another negative trend in our study was that there are some underweight children from the three degrees as the values ranged from $10.8 \%$ for the 6-year-old girls (control group) up to $27.5 \%$ for the 5 year old boys from the experimental group.

In conclusion, we would like to underline that overweight and obesity are an important medical and social problem escalating the last years. However, we should have in mind the other extreme trend - the existence of underweight children - II and especially III degree, so our efforts should be to directed towards regulating the body weight in two directions - both reduction or increasing in dependence of any individual case.
\end{abstract}

Key words: Preschool age, BMI, Physical activity

\section{INTRODUCTION}

Preschool age is an important period in human development. Most of the authors define it as basic period regarding the physical development of individuals and the proper period for building of the basic motor skills and habits $(1,2)$. As it is known, physical activity at that age is of a great importance for the psycho-physical development of man and effects the next periods in his ontogenesis (3, 4).

Analysing various information sources as well as pedagogical practice, we came to the conclusion that there is a certain contradiction

\footnotetext{
Correspondence to: Chief assistant Tsveta Traykova, National Sports Academy „Vasil Levsky", Theory of the physical education Department, 1700, Sofia, Studentski grad, CvetaDimitrova@abv.bg, Mobile:0896630163
}

between the need of organized and spontaneous physical activity of preschool children in kindergartens on one hand and the use of new, appropriate and attractive means and methods for encouraging them to participate in various forms of physical activities in the kindergarten on the other.

One of the main indicators for the assessment of physical development is Body Mass Index (BMI) $(5,6)$. A number of studies have shown that the higher levels of this index in childhood would have a negative effect in the following years in the life.

The aim of our study was to establish the dynamics of BMI of preschool children as a result of an enriched physical activity program in physical culture. 


\section{METHODOLOGY}

The subject of the study was the physical development of 5-7 years old children from a preparatory group for school.

The object was the state of BMI of children of that age group.

208 children from two kindergartens and preschool education groups in Sofia Schools were investigated - 113 boys and 95 girls divided into two groups - experimental and control. The experimental group followed a new physical activity program created by the author, while the control group followed the standart physical culture program. (author dissertation). The studied subjects were tested twice - at the beginning and at the end of the pedagogical experiment. Two main anthropometric parameters were measured height and body weight and the Body Mass Index was calculated by the standard formula
TRAYKOVA TS. BMI $=($ weight $(\mathrm{kg})) /($ height $(\mathrm{m} \wedge 2)$. The results of our research were compared with the scale, developed by T. Cole et al. (2000, 2007), which presents BMI in six levels, namely: obesity, overweight, normal weight, underweight I-st degree, II-nd degree and IIIrd degree $(7,8,9,10)$.

The significance of this index is that it determines the level of fatness of the body and is accepted as a criterion for assessing the nutritional status of a person (7).

The following methods were used: theoretical analysis of literature, pedagogical experiment, anthropometry. graphic analysis and statistical methods.

\section{RESULTS AND ANALYSIS}

The calculated mean values of BMI for the third age experimental and control groups of boys and girls are presented in Figure 1.

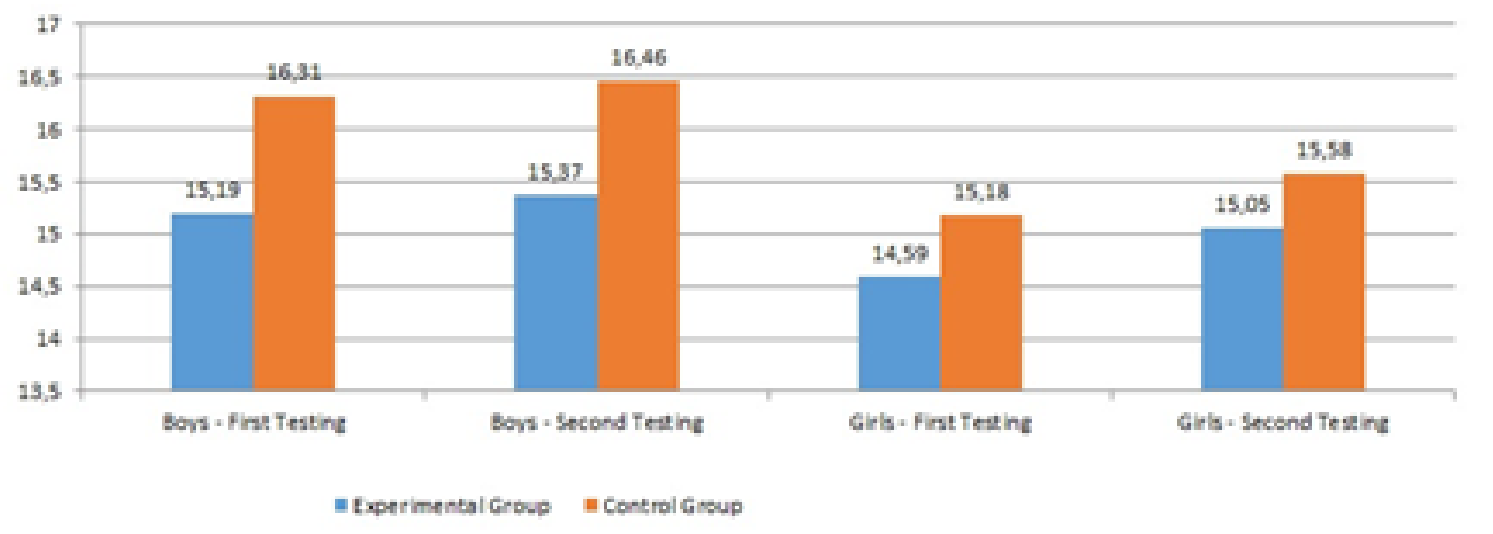

Figure 1. Mean values of BMI for the third age experimental and control group - boys and girls

The mean values from the first measurement of boys are 15.19 for experimental and 16.31 for the control group respectively. In the second measurement, they were 15.37 and 16.46, respectively. In both studies the control group has higher values of Body Mass Index, but generally all the studied boys are within the normal range of that index.

In the girls of the same age it was found that the mean values of BMI of both experimental and control groups were again in normal limits. The higher values of BMI are recorded in the control group too, which in the first measurement was 15.18 and in the second -15.58 .

The following mean values of BMI for the fourth age group - boys and girls - are presented in Figure 2.

Both in the first and the second measurements the control group of boys has higher values of Body Mass Index -16.58 and 16.51, respectively. The experimental group shows results of 15.57 in the initial and 15.88 in the final testing. As a whole, all the boys studied from the fourth pre-school age group did not exceed the limit values for normal Body Mass Index.

Figure 2 illustrates the results of girls of the same age too. It is evident that the girls from the experimental group - first and second testing have higher BMI values respectively 17.13 and 17.28 at the final. The control group has a lower values of BMI but all the studied persons are in the normal limits of that index. However, we should note that the result of the second measurement in the experimental group - 17.28 is approaching the upper limit of the normal value (17. 4). What's important here is that sexual differences were manifested in all groups as the boys have higher results concerning the normal values of BMI. 


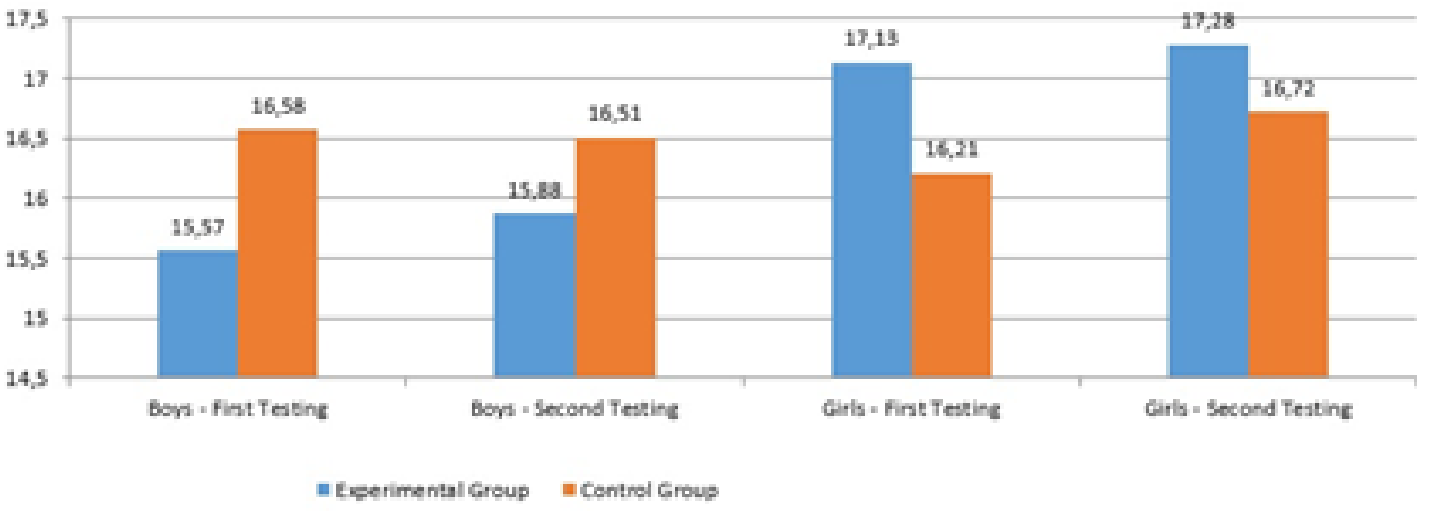

Figure 2. Mean values of BMI for the fourth age experimental and control group - boys and girls

BMI values increased a little at the end of the experiment in both boys and girls from the third and fourth age groups as the differences between the first and the second measurement in experimental and control group - boys and girls - are with high statistical significance ( $p$ $<0.05$ ). Probably these results are due to the bigger increase in body weight in comparison with the height.
In order to reveal the distribution of BMI values in percentage we used the scale developed by T. Cole et al., already noted as six levels: obesity, overweight, normal weight, underweight I-st degree, underweight II-nd degree and underweight III-rd degree.

Figure 3 presents the frequency of all categories of BMI in boys of the third preschool group.

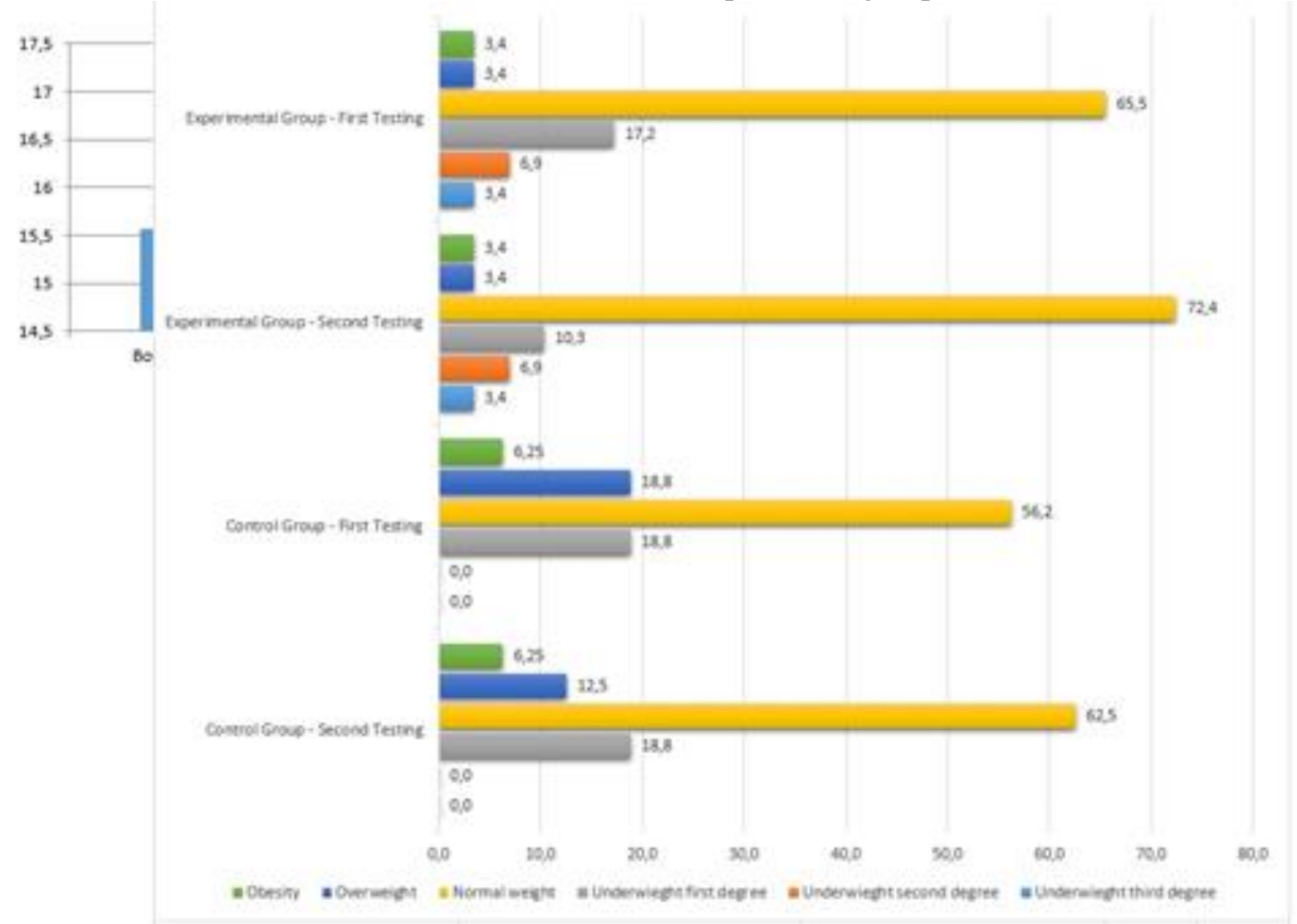

Figure 3. Frequency of all levels of BMI in the third preschool group - boys (in \%)

As it is seen from the figure the boys from the experimental group with normal values of BMI in the first testing are $65.5 \%$ which increase up to $72.4 \%$ in the second one. The percentage of the boys who are overweight and obese is 6.8 and of those who are underweight in all the three degrees is 27.5 in the initial testing.
In the boys of the control group normal BMI have $56.20 \%$ at the initial testing and $62.50 \%$ at the final one. It is also recorded that $6.25 \%$ of the children are with obesity (first and second testing) and $18.8 \%$ (at first testing) are overweighed. 
The boys who are underweight I-st degree are $18.8 \%$ in both measurements. No boys who are underweighted II-nd and III-rd degree were registered.

It is interesting to mention that the number of the children with normal BMI increased at the end of the experiment. The overweight boys from the control group and the underweight ones from the experimental group decrease in number / in \% /. In all other BMI categories no changes are observed.

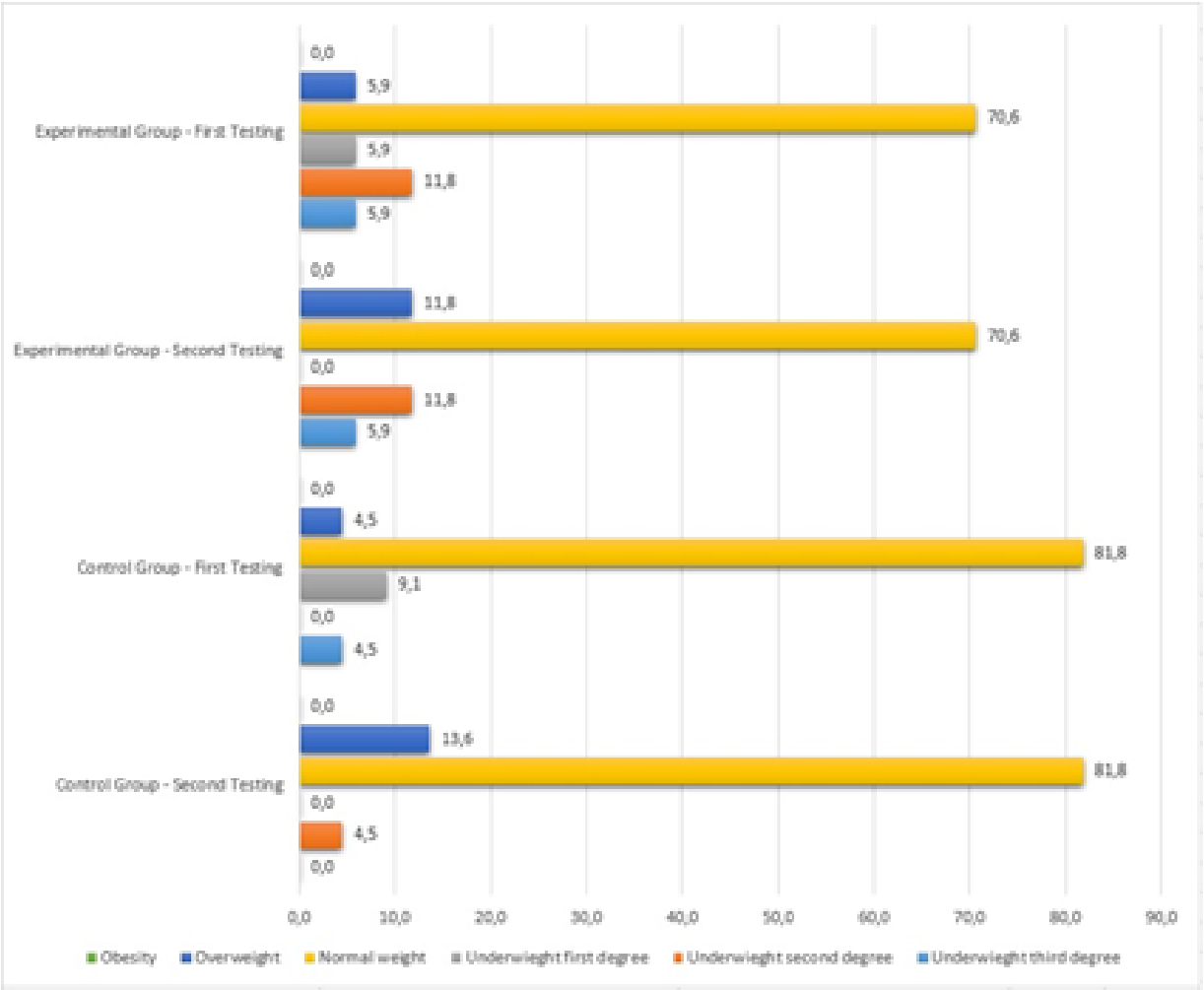

Figure 4. Frequency of all levels of BMI in the third preschool group - girls (in \%)

The girls in both experimental and control groups of the same age (Figure 4), have normal values of BMI as follows $-70.6 \%$ in both measurements of the experimental group and $81.8 \%$ of the control one. No change was registered at the end of the experiment.

Overweight girls are $5.9 \%$ in the first measurement of the experimental group and increase up to $11.8 \%$ in the second measurement. An increase of the overweight girls of the control group is establish in the second measurement - from 4.5 to $13.6 \%$. In the category underweight I-st degree and underweight II-nd degree in first measurement are registered $5.9 \%$ of the girls of the experimental group and $9.1 \%$ of the control one. While in the second measurement the values are $11.8 \%$ in the experimental group and $4.5 \%$ in the control one as in the experimental group the percentage of underweight girls is increased.
The results of the boys in the fourth age group are presented in Figure 5.

The percentage of children from the experimental group with normal BMI is high $81.3 \%$ in the first measurement and $84.4 \%$ in the final one. In the control group that percentage is lower $-65.8 \%$ at the beginning and $68.6 \%$ at the end of the experiment. The boys which are overweight are $3.1 \%$ and their number doesn't change during the experimental period. In the control group the percentage is higher $-22.8 \%$ and marks a little decrease in the overweight boys $-20.0 \%$. As shown in the figure the underweight boys of the experimental group are $9.4 \%$ and their number decrease to $6.3 \%$ in the second measurement. The percentage of the underweight control group recorded in both measurements is one and the same $-11,4 \%$. 
TRAYKOVA TS.

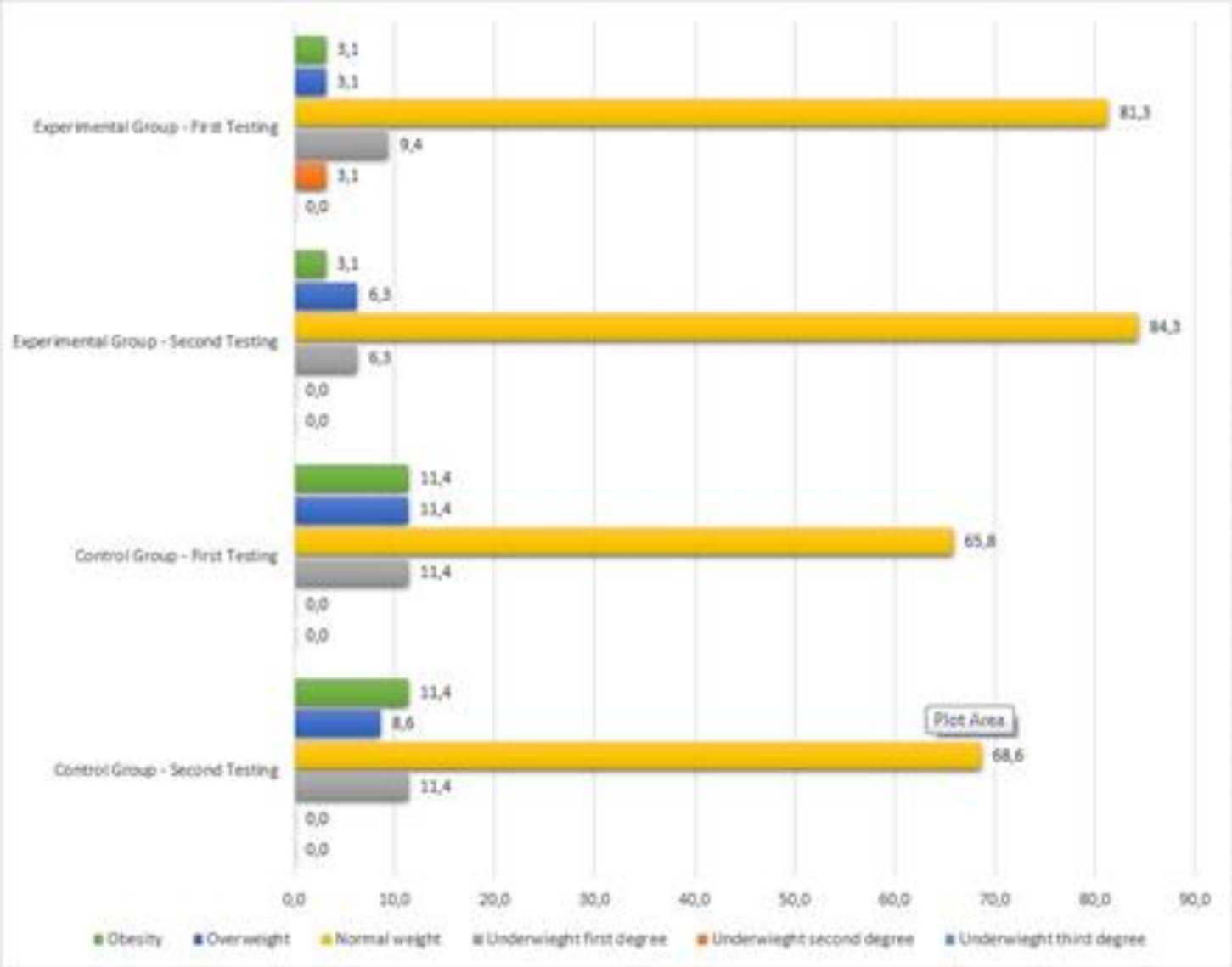

Figure 5. Frequency of all levels of BMI in the fourth preschool group - boys (in \%)

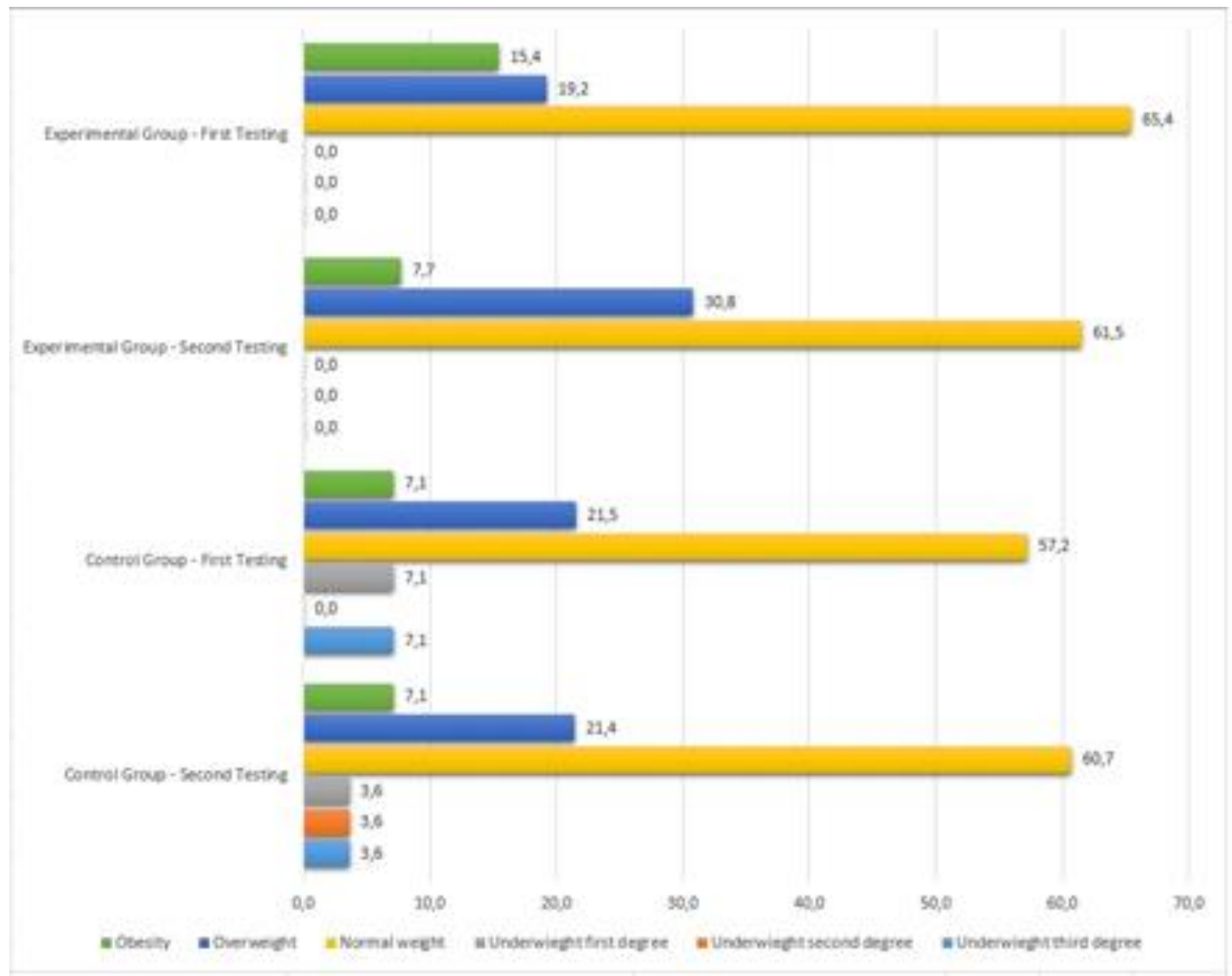

Figure 6. Frequency of all levels of BMI in the fourth preschool group - girls (in \%) 
The girls of the experimental group of the same age (Figure 6) have normal BMI values which in the first measurement are $65.4 \%$ and in the second $-61.5 \%$. so they decreased a little. In the control group, the percentage of the children with normal BMI is $57.2 \%$ which is increased at the end up to $60.7 \%$.

The percentage of the overweight and obese girls of the experimental group is $34.6 \%$ in the initial measurement and increases up to $38.5 \%$ in the final measurement. It should be mentioned that the percentage of the obese girls decrease while that of the overweight increase. An interesting fact is that is that the percentage of the girls with overweight and obesity increase with age.

The percentage of 21.4 is recorded for the overweight girls of the control group which didn't change at the end. The obese girls at the beginning and the end of the experiment are $7.1 \%$. So the girls of the control group with overweight and obesity have lower percentage of these categories.

In the control group $7.1 \%$ was registered as underweight I-st degree in the first measurement, which decreased to $3.6 \%$ in the second one. With the same percentage are presented the girls which are underweight II-nd degree.

\section{CONCLUSION}

The detailed analysis of the results of the BMI of 5-7 years old investigated children gives us the ground to make the following conclusions: *The mean values of Body Mass Index of boys and girls from the two age groups increased significantly at the end of the experimental period.

*The investigated children from both sexes and all age groups - experimental and control have the highest percentage of BMI in norm values.

*A high percentage of children with overweight and obesity is also found in all groups.

*Another negative trend is observed as a result of our study - about $1 / 4$ th of the children are underweight.

* Sexual differences were manifested in all groups as the boys have higher results in the normal values of BMI.

Our study shows that BMI is influenced by many other internal and external factors independently of the different forms of physical activity, which were not an object of our investigation.

In conclusion, we would like to underline that overweight and obesity are an important social and medical problem escalating in the last years (11). However, we should have in mind the other important trend - the existence of underweight children whose number increases. So our efforts should be directed towards regulating the body weight in two directions both reduction or increasing it in dependence of any individual case.

\section{REFERENCES}

1. Gyurov, D., etc. Program system for preparatory group in kindergarten and at school. Hand in Hand. Prosveta, C., 2003.

2. Damianova, M. Author of the chapter "Physical Culture" in the book for teachers MOLIVKO about preschool education and preparation of 5-6 years old children. SPEECH, Veliko Tarnovo, 2010.

3. Traykova, Tsv. State of physical development and motor abilities of children attending preparatory group for school (5 - 7 years old), Dissertation,2018.

4. Slanchev, P., etc., Physical development, physical fitness and neuro-psychological reactivity of the Bulgarian population. III rd National Survey (1980-1982), NSA "V.Levsky", 1992.

5. Slanchev, P., Sports medicine. A textbook for students at the NSA, 1998.

6. https://bg.wikipedia.org/

7. Dyakova,G., Impact of motor activity on harmful habits and physical fitness of students. Monograph, ISBN 978-954-938358-4, 2010.

8. Physical development of children and youths in Bulgaria on the borderline between 20th and 21st century, edited by Corr. Mem. Yordan Al. Yordanov, DD,PhD, DSc, 2012.

9. Cole, T., M. Bellizzi, K. Flegal, W. Dietz. Establishing.a Standard Definition for Child Overweight and Obesity Worldwide: International Survey. British Medical Journal, 2000, 320, 194-201.

10. Cole, T., Flegal, K., Nicholls, D., Jackson.A., Body Mass Index Cut offs to Define Thinness in Children and Adolescents: International Survey, British Medical Journal, 335, 194201, 2007.

11. Toppuzov, IV. Obesity in adolescents distribution, characterization and treatment approach. Dissertation, C., 1987. 